\title{
PERSPECTIVE
}

\section{Una Extranjera en el Extranjero}

\author{
Amber N Himmler \\ Panamerican Journal of Trauma, Critical Care \& Emergency Surgery (2021): 10.5005/jp-journals-10030-1319
}

"Global surgery" is a term used in academia almost in the same vein as fellowship-trained subspecialties, i.e., "Trauma Surgery" or "Minimally-invasive Surgery". After spending a year in Ecuador learning and living a global experience, this compartmentalization truly neglects to express how beautifully variable and nuanced this particular practice of surgery can be. Beyond the intricacies of science, it encompasses a unique, intangible human element. I have learned that the practice of global surgery is centered around the development of lasting relationships and truly understanding the context in which one is living and working. I gained an incredible amount of knowledge and clinical experience in 1 year abroad. Yet, nothing can be experienced in a vacuum. I was welcomed into the lives of people who were previously strangers and quickly became close friends and mentors. Alongside them, I experienced everything from funerals to baby showers, from protests and riots to holiday parties and carnaval. Only after spending late nights rushing to eat a seco de pollo with colleagues in-between cases, and long hours sifting through dust-ridden stacks of medical records am I even starting to understand what it means to be a global surgeon.

This is not to say there is not a significant amount of discomfort involved. I had completed 3 years of general surgery training before my research year but despite that, after arriving I found myself feeling unsure of the next step, often feeling more awkward than the student members of the team. Despite having studied Spanish up to that point, I struggled with not having the words to ask for surgical instruments or certain lab tests, never mind presenting a complex patient in the intensive care unit. Moreover, without a respiratory therapist to set up a ventilator, a scrub tech to assemble laparoscopic instruments, or a circulating nurse to ensure that the count was correct, I quickly was overwhelmed by how much I had to learn to be able to work in this environment.

Four to five months passed before I felt accepted by the residents and attendings and adapted to the system in which I was working. By the end of 2019, I was able to write progress notes and operative notes in Spanish, tie my own endoloop knots, and knew the best spots for an afternoon bolón or tigrillo. I learned different approaches to common surgical problems such as the open appendix or a bile duct exploration, with which I had almost no experience in the United States. I became more aware of every supply needed to complete an operation while having to open my own back table, and even more aware of how much each supply was really needed when a patient's family member had to be sent to purchase it. I fell asleep on the stretchers alongside the surgical residents who had, after all of this time, become close and trusted friends. I had learned where in the hospital I needed to search for each individual piece of data. I became more efficient in translating scientific ideas from Spanish to English, and I had some successes in helping my colleagues publish their work.
Department of Surgery, Medstar Georgetowan University Hospital and Washington Hospital Center, MedStar Georgetown University Hospital, Washington, DC, USA

Corresponding Author: Amber N Himmler, Department of Surgery, Medstar Georgetowan University Hospital and Washington Hospital Center, MedStar Georgetown University Hospital, Washington, DC, USA, Phone: +91 987485889, e-mail: amberhimmler@gmail.com

How to cite this article: Himmler AN. Una Extranjera en el Extranjero. Panam J Trauma Crit Care Emerg Surg 2021;10(2):87-88.

Source of support: Nil

Conflict of interest: None

On reflecting on my time abroad, I realize that I am only starting to fully understand what it means to be a global surgeon. The term "global" is a misnomer. Every experience, hospital, and country will be different. The unifying message of global surgery is that one must learn and take into account the context in which one is working to effectively care for patients in a given community, or even a given setting. This may look different in Ecuador than in other countries, but also within communities or hospitals that may be separated by only a few kilometers. The exciting part of global surgery is that, while the practice of surgery may not change much, one has the opportunity to adapt their practice to the microcosm into which one has landed.

High-income countries (HICs) stand to learn a lot from the experiences in low-and-middle-income (LMIC) countries. The dissemination of information is undeniably reliant upon publications. It did not take long for me to realize why there is such a substantial publication gap among Latin American surgeon scientists. In the absence of work-hour restrictions and with a shorter overall period to accomplish their training, residents find it nearly impossible to fit research into their careers at the early stages. Attendings and professors at the university rarely, if ever, have dedicated time in their schedules or stipends dedicated to research, and most surgeons have to work in both the public and private sectors to make enough money to make a living. Hospital administration can be hard-pressed to prioritize research when nursing staff and basic supplies are often lacking due to insufficient or irregular sources of funding. Some journals require a fee that can be impossible to pay out-of-pocket, even at reduced rates, in the absence of an institution able to cover that cost. In research, there is significant value-added for trainees from HICs. With a robust research infrastructure at their fingertips, perhaps those in HICs should focus on assisting LMIC neighbors in sharing their knowledge with the rest of the surgical community. 
A year of living in global surgery has altered profoundly my understanding of global surgery. No amount of planning or preparation could have primed me for the challenges and the growth that I experienced during this time. I realize now that I have only scratched the surface of this new and ever-evolving field of surgery. Through this experience, I now have colleagues who are on their way to becoming surgeons. Laying down the roots for these life-long relationships was a critical first step in what I hope to be a long and fruitful career in global surgery. 